\title{
Network Traffic Obfuscation and Automated Internet Censorship
}

\author{
Lucas Dixon Thomas Ristenpart Thomas Shrimpton \\ Jigsaw, Cornell Tech, University of Florida
}

\begin{abstract}
Internet censors seek ways to identify and block internet access to information they deem objectionable. Increasingly, censors deploy advanced networking tools such as deep-packet inspection (DPI) to identify such connections. In response, activists and academic researchers have developed and deployed network traffic obfuscation mechanisms. These apply specialized cryptographic tools to attempt to hide from DPI the true nature and content of connections.

In this survey, we give an overview of network traffic obfuscation and its role in circumventing Internet censorship. We provide historical and technical background that motivates the need for obfuscation tools, and give an overview of approaches to obfuscation used by state of the art tools. We discuss the latest research on how censors might detect these efforts. We also describe the current challenges to censorship circumvention research and identify concrete ways for the community to address these challenges.
\end{abstract}

\section{INTRODUCTION}

Around the world, well-resourced governments seek to censor the Internet. Censorship is driven by the desires of governments to control access to information deemed politically or socially sensitive, or to protect national economic interests. For example, in China performing Internet searches for information on Tiananmen square reveals no information about the events in 1989, and communication platforms run by companies outside China, such as GMail and Twitter, are among those that are routinely blocked. Although China's "Great Firewall" is perhaps the best known example of Internet censorship by a nation-state, similar controls are enacted in Turkey, Iran, Malaysia, Syria, Belarus and many other countries 1

In this article we will focus on the technical underpinnings of automated censorship and of the network traffic obfuscation tools aimed at circumventing it. By automated censorship, we mean government deployed or mandated network monitoring equipment that is typically installed at Internet service providers (ISPs). These systems detect and disrupt the flow of censored information without any direct human involvement and are applied broadly to enforce government policies over all citizens.

\footnotetext{
${ }^{1}$ An excellent survey of the censorship policies by various governments around the world is provided by the OpenNet Initiative https://opennet.net//
}

This differs from targeted approaches to taking down Internet content, such as the United States government's take-down of the Silk Road, which was an Internet marketplace for illicit goods such as drugs and weapons.

We will first review how modern deep-packet inspection (DPI) devices enable censors to filter traffic based on the application-layer content they contain, e.g., search terms, requested URLs, filetypes, and information that identifies the application itself. We will then explore the technical methods used to obfuscate application-layer content, in order to minimize the usefulness of DPI. These make intimate use of cryptography, though in nonstandard ways. We place the methods into one of three categories: those that randomize every bit of packet payloads, those that transform payloads so that they mimic typical "benign" traffic (e.g., HTTP messages), and those that tunnel payloads through actual implementations of network protocols (e.g., a VPN or HTTPS proxy). Each category comes with its own set of assumptions about the censor's approach, and targets a different point in the security/performance design space.

We then discuss the challenges to progress faced by researchers in this area. Chief among these is the dearth of solid information about the technical capabilities of real-world censors, and their sensitivities to filtering errors (both missing targeted traffic and blocking nontargeted traffic). Similarly, we lack understanding about real-world users of censorship-circumvention tools. In both cases, even gathering such information presents significant challenges, as we will see.

The final contribution of this article is to suggest concrete tasks and research efforts that would give considerable aid to the design of future obfuscation tools.

\section{BACKGROUND: FROM CENSORSHIP TO PROTOCOL OBFUSCATION}

In what follows, we will use terms like "censor", "adversary" and "sensitive flow" repeatedly. To aid understanding, we provide a summary of such key terms in Fig. 1

The technical means of automated censorship assumes that the censor enjoys privileged access to network infrastructure. Governments typically have this via direct 


\begin{tabular}{ll}
\hline $\begin{array}{l}\text { Sensitive } \\
\text { flow }\end{array}$ & $\begin{array}{l}\text { Network communications targeted for being } \\
\text { offensive, counter-regime, or using obfuscation } \\
\text { tools }\end{array}$ \\
$\begin{array}{l}\text { Censor, } \\
\text { Adversary }\end{array}$ & $\begin{array}{l}\text { The party that controls the communication } \\
\text { network and is attempting to identify and } \\
\text { restrict sensitive flows of information }\end{array}$ \\
$\begin{array}{l}\text { Obfuscation } \\
\text { tool }\end{array}$ & $\begin{array}{l}\text { Software designed to prevent censors from } \\
\text { identifying/blocking communications }\end{array}$ \\
$\begin{array}{l}\text { Background } \\
\text { traffic }\end{array}$ & $\begin{array}{l}\text { Non-sensitive flows coexisting (potentially) with } \\
\text { sensitive flows on the network }\end{array}$ \\
$\begin{array}{l}\text { Collateral } \\
\text { damage }\end{array}$ & $\begin{array}{l}\text { Background traffic adversely affected by } \\
\text { censor's efforts to control sensitive flows }\end{array}$ \\
Proxy & $\begin{array}{l}\text { Party who will forward traffic to broader } \\
\text { Internet on behalf of censored client; external to } \\
\text { censor-controlled network }\end{array}$ \\
\hline
\end{tabular}

Fig. 1. Summary of often-used technical terms.

or indirect control of Internet service providers (ISPs). In the security community this is called an on-path adversary: it is on the path of network packets between the user seeking to access sensitive content, and the provider of that content (e.g., a web server).

\section{A. Traffic Identification Methods}

Network censors face two main tasks: to accurately identify network traffic that carries or attempts to access sensitive content, and (potentially) to perform a censoring action on that traffic. Examples of the latter are dropping packets to degrade the user's experience, forcing one or both of the endpoints to drop the connection (e.g. by sending TCP reset messages), and redirecting web connections to a censor-controlled web server. But as censoring actions must be preceded by identification of targeted traffic, we focus only on the censor's identification task.

Address-based identification. Originally, attempts to access censored Internet content were identified by first associating addressing information - IP addresses and port numbers, domain names - with sensitive content, resulting in a blacklist of addresses. Any network connections to these addresses were deemed as attempts to access sensitive content. For blacklisted domain names, the censor can direct ISPs to run modified DNS software. Connections to these domains are then typically blocked or misdirected. For IP addresses, the censor can install hardware mechanisms at ISPs that compare information in a packet's IP header against the list. As the IP headers appear early in the packet, one needs only to perform "shallow" packet inspection, disregarding information encapsulated deeper in the packet. TCP or UDP port information is similarly available via shallow inspection.

A user can avoid both domain-name- and IP/portbased identification by using a proxy, a cooperative

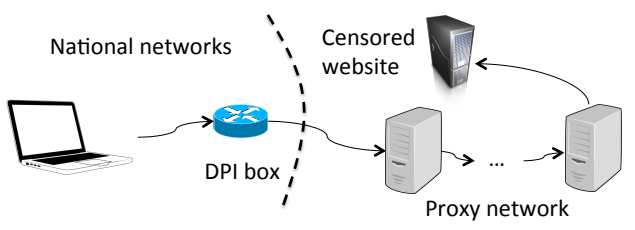

Fig. 2. Proxy-based circumvention of censorship. A client connects to a proxy server that relays its traffic to the intended destination. It may send the traffic through multiple proxies before the destination should anonymity be desired.

machine whose address information is not blacklisted. The operation of proxy-based circumvention is summarized by the diagram in Fig. 2. Many tools such as Tor, uProxy, Anonymizer, and Psiphon rely on the use of proxies ${ }^{2}$ Recently, domain fronting [6], a form of decoy routing [8], [10], [20] that operates at the application layer, has also been successfully deployed and is discussed further in Sec. III]

Identification via deep-packet inspection. The success of proxy systems in practice has led censors to deploy new DPI mechanisms that identify traffic based on information deeper inside the network packets. For example, application-layer content carried in (unencrypted) packet payloads can divulge user-generated data, such as keywords within search URLs. We already noted China's Great Firewall blocking traffic that contains blacklisted keywords.

In reaction to DPI, modern circumvention tools systems encrypt content to proxies, preventing this kind of keyword search. The result has been that sophisticated modern censors now use DPI to attempt to identify and block traffic created by circumvention tools. For example, China used DPI to detect Tor connections by looking for a specific sequence of bytes in the first application-layer message from client to server. This sequence corresponds to the TLS cipher suites that were selected by Tor clients, which were unlike those selected by other TLS implementations. As another example, Iran used DPI to determine the expiration date of TLS certificates. At the time, Tor servers used certificates with relatively near expiration dates, while most commercial TLS servers chose expiration dates that are many years away.

These blocking policies used fairly sophisticated information about the circumvention tool - the particular implementation of TLS - being carried in packet payloads. But even a crude scan of application-layer content can discover that standard protocols (e.g. SSH, TLS) are being transported. This may be enough to trigger insensitive blocking, or logging of source/destination IP

${ }^{2}$ These tools are available online at: https://torproject.org https:// www.uproxy.org https://anonymizer.com https://psiphon3.com 
addresses for more detailed analysis or later action.

\section{B. Network Traffic Obfuscation}

The use of DPI to perform tool identification motivated the circumvention-tool community to seek countermeasures, culminating now in an entire area of research and development: network traffic obfuscation. The goal is to force DPI-using censors to abandon attempts at protocol identification, because accurate identification at-scale requires prohibitive resources. For example, by developing tools whose traffic can reliably force DPI to misclassify the traffic as that of a non-blacklisted tool. It is network traffic obfuscation that we will concern ourselves with in the remainder of this paper.

We want to emphasize that our focus on obfuscation does not belie the importance of other aspects of censorship circumvention. IP- and DNS-based filtering are still more prevalent than DPI-based mechanisms, and circumventing such filtering remains a challenge in practice. Ultimately circumvention requires a variety of mechanisms that work in concert to defeat a censor and a system is only as good as its weakest link.

Network traffic obfuscation threat-models. A threat model is a particular combination of censor capabilities and goals. We will provide only informal description of threat models for the purposes of this article. We emphasize that the threat models presented here apply only to gathering information from network flows (IP information, application-layer content, etc.), and not to censoring actions (blocking or otherwise disrupting the communication) that this information enables. Information may be gathered by using passive or active techniques.

A passive technique simply observes traffic flows. One high-profile example of passive techniques occurred during the February 2012 anniversary of the 1979 Iranian revolution. The Islamic Republic of Iran mounted a large-scale censorship campaign, blocking various IP addresses associated with anti-censorship tools such as torproject.org, and using DPI-based identification of TLS network flows 3

An active technique modifies existing packet contents or injects completely new packets. Examples of active attacks that manipulate connections by modifying or dropping specific packets have appeared in the academic literature [7]. While far less common than passive techniques, active techniques have been used by the Chinese government in order to identify Tor proxies [18]. We give a list of example passive and active attacks in Fig. 3 . For brevity, in the remainder of the paper we focus primarily on passive attackers.

${ }^{3}$ See: https://blog.torproject.org/blog/ iran-partially-blocks-encrypted-network-traffic
So far we have spoken mostly about the capabilities part of the threat model. In terms of the goals, the typical assumption is that censors minimally want to accurately classify traffic as either targeted (for application of policy) or not. Accuracy has two facets. The first is a high true-positive rate, meaning that the fraction of targeted traffic that is marked as such is close to one. The second is a low false-positive rate, meaning that the fraction of non-target traffic incorrectly marked as targeted is close to zero. Obfuscation tools are often said to be unobservable if they can cause sufficiently low true-positive and high false-positive rates. What constitutes sufficient error rates, however, is largely unknown. However, tools with the potential to induce high falsepositive rates are preferred as it is argued that censors will be less likely to block them. Let us say more about why.

Sensitivity to collateral damage. Collateral damage occurs when a censor's detection mechanisms suffer from false-positives, leading to mislabeling network flows as associated to a blacklisted tool, protocol, or content when, in fact, the flow is not. Why should censors be sensitive to collateral damage? The answer is highly context dependent, varies over time, and is ultimately a question of socio-political factors. The example of the February 2012 censorship campaign by Iran is an example of insensitive censorship (since the vast majority of TLS traffic was almost certainly not used for circumvention or bound for hosts deemed offensive by the regime). We note that this particularly broad censorship policy lasted for only a short period of time, and that it is is typical for censorship policies to change, in terms of targets and sensitivity, over time. We will discuss later how measuring sensitivity remains a critical open research challenge.

\section{OBfuSCATION APPROACHES}

Use of DPI by censors has inspired researchers and activists to explore a variety of countermeasures aimed at obfuscating packet payloads. We break down approaches into four categories: encryption, randomization, mimicry, and tunneling.

Encryption. A natural first thought is that conventional encryption is already a good obfuscator. Indeed, encryption renders unintelligible the data that is encrypted. But most encryption protocols have plaintext headers in each packet payload that identify the protocol itself, and sometimes indicate the particular application that is implementing the protocol. We've seen in Iran and China that plaintext headers contain fingerprintable parameters that can help a censor distinguish between, say, Tor's implementation of TLS from non-Tor implementations. In short, just encrypting data does not guarantee a level 


\begin{tabular}{|l|l|l|l|}
\hline Country & Dates & Techniques and Targets & Passive/Active \\
\hline China & $2007-$ & IP/DNS blacklisting, Tor TLS header fingerprinting, Tor-relay handshake probe \\
Iran & Feb. 2012 & IP blacklisting, keyword flagging, TLS handshake fingerprinting \\
Turkey & Early 2014 & IP/DNS blacklisting, BGP hijack of Twitter and Youtube \\
Pakistan & Intermittent & IP/DNS blacklisting, URL, keyword, and filetype flagging \\
Syria & $2011-$ & IP/DNS blacklisting, instant message fingerprinting, social media keyword blacklisting \\
Ethiopia & 2012 & Skype detection, Tor TLS fingerprinting & Poth \\
Belarus & $2014-$ & IP/DNS blacklisting, filtering according to packet content \\
Kazakhstan & 2012 & Encryption fingerprinting, Tor TLS handshake fingerprinting \\
Egypt & $2014-$ & IP/DNS blacklisting, instant message fingerprinting, social media keyword blacklisting & Passive \\
\hline
\end{tabular}

Fig. 3. Examples of censorship by nation states. Techniques and targets in bold rely on DPI.

of unobservability sufficient for the goal of making DPI unreliable for censors.

Randomization. If conventional encryption, like plaintext protocols, can leave exploitable fingerprints for DPI, why not attempt to randomize every byte in the packet payload? This strategy has been described as attempting to make network flows "look like nothing" by the obfs family of obfuscators [14], and is a strategy also used by Dust [17] and ScrambleSuit [19]. ScrambleSuit and the obfs methods are designed specifically for the Tor pluggable transport framework called "Obfsproxy".

The standard approach to randomizing a payload is to apply a stream cipher to every byte. Assuming a passive censor that does not have access to the stream cipher secret key, this approach makes protocol identification difficult because there are no fixed fingerprints to observe. Because stream ciphers do not provide the strong confidentiality and authenticity guarantees sought, they are typically applied on top of modern encryption methods that do.

Underlying the use of randomizing, "look-likenothing" obfuscators is the assumption that censors use only blacklisting: they allow all traffic except that which conforms to an explicit set of disallowed traffic types. If censors use whitelisting, exclusively or in addition to blacklisting, then fully randomized payloads will not avoid censorship. This is by virtue of looking like no actual network traffic.

Mimicry. To address whitelisting censors, a class of obfuscation methods attempt to evade censorship by masquerading as a whitelisted protocol. Loosely, rather than trying to look like nothing, mimicry-based obfuscators try to make packet payloads look like something that they are not, but that will be identified by DPI as allowable. A common example is to make payloads look like HTTP, which is rarely blocked because of its ubiquity. Mimicry in the context of anti-censorship originates with the Tor project prepending HTTP headers to Tor packets [14], though mimicry can be thought of as steganography against a particular type of adversary (DPI systems). Stegotorus [16] and SkypeMorph [13], for example, attempted to use steganographic approaches to look like HTTP and Skype traffic, respectively. Un- fortunately they have prohibitively poor performance.

A minimalist but flexible approch to mimicry can be found in format-transforming encryption (FTE) [11]. As implemented in Tor, uProxy and elsewhere, FTE allows programmers to use regular expressions to specify the format of ciphertexts. Because regular expressions are also used by DPI, one can often precisely force misclassification of the protocol.

Mimicry obfuscators attempt to make packet payloads look like a particular "cover" protocol, but do not actually use an implementation of the targeted cover. This means that the syntax and semantics of messages emanating from mimicry systems may deviate, often significantly, from that of messages conforming to the cover protocol. As one example, a mimicking obfuscator may produce a message purporting to be an HTTP GET after having just received a message purporting to be an HTTP GET, thereby violating proper HTTP semantics.

Tunneling. Instead of custom obfuscation implementations of mimicry that reproduces some aspects of the cover protocol, tunneling obfuscators transmit messages produced by an actual implementation of the cover protocol. Tunneling is of course not something new to obfuscation: all proxy systems use tunneling over encrypted channels. So any VPN or HTTPS proxy might be considered a tunneling obfuscator. The key difference is that tunneling obfuscators are meant to obfuscate that an anti-censorship tool is being used, and sometimes even obfuscate that encryption is being used. A VPN or proxy may not (attempt to) hide such facts.

A notable example of a tunneling obfuscator is the Tor pluggable transport Meek [6]. Meek tunnels via TLS to HTTP load-balancers operated by Google, Amazon CloudFront, or Microsoft Azure. This is referred to as "domain fronting" because the ostensible domain revealed at the TCP layer (and so, in the clear) is google.com (say), but the underlying encrypted request is for a domain that routes to a Meek-enabled Tor relay 4 As with Tor-TLS, the Meek obfuscator is at pains to ensure that tunneled Tor-connections to the load balancer look like conventional TLS connections to

\footnotetext{
${ }^{4}$ Interestingly, a similar use of domain fronting was employed by the GoAgent system and was popular in China in 2013.
} 
the load balancer. It's worth noting that this approach relies on an undocumented "feature" of load-balancer implementations, and it is conceivable that companies may modify future load balancers in a way that prevents domain fronting.

The table appearing in Fig. 4 summarizes a range of obfuscators that have been deployed and compares them qualitatively in terms of deployment, how keys are exchanged (should encryption be used to perform obfuscation), and performance. It also includes a summary of attacks that have been explored against them, as discussed in Sec. IV.

\section{Seeing Through Obfuscation}

Can real censors detect obfuscators? Researchers have begun preliminary investigations into this question, and we will summarize their approaches here focusing on passive techniques. The takeaway message from what follows is that the answer is not yet clear.

Syntactic and semantic mismatch. The first intensive investigation into obfuscator detection is the work of Houmansadr, Brubaker, and Shmatikov [7]. They focus on mimicry systems in particular, and claim that all such systems are fundamentally flawed because they will never perfectly replicate all aspects of a target protocol implementation.

They look specifically at the extent to which messages emitted by currently implemented systems abide by the proper syntax or semantics of the cover protocol. By syntax we mean that the emitted messages are parsable as cover protocol messages - required headers are in the right places, the correct character sets are used, etc. Semantics refers instead to whether messages are meaningful relative to the proper functioning of the protocol.

Most mimicry systems explicitly made no attempt to mimic all aspects of the cover protocol, making the search for syntactic and semantic mismatches a foregone conclusion. As examples, Stegotorus doesn't implement most of HTTP, and the packets sent when using FTE (in a way that favors performance) contains large numbers of random, unformatted bits. The authors show how to take advantage of such mismatches. In particular, they detail a number of ways to detect Stegotorus, SkypeMorph, and CensorSpoofer. None of these obfuscators are actually in use (although for reasons unrelated to security), and so these attacks were only tested against simulated stand-ins for deployed systems. It is possible that real deployments would be more (or less!) vulnerable to their attack methods.

We also note that the authors did not measure the collateral damage of their tests, i.e., to what extent the attacks they develop would misclassify benign coverprotocol traffic as obfuscated. Subsequent work [15] showed that some of their tests would lead to a false positive rate of $37 \%$ if naively deployed, while others appear effective at avoiding collateral damage.

Entropy tests. The semantic and syntactic checks above will not help one identify the use of randomizing obfuscators, as these explicitly try to look like no actual protocol. Wang et al. [15] evaluated the use of efficient entropy tests in the context of detection of randomizing obfuscators. Their techniques focus on a small window of bytes occurring in the first few messages of an observed flow. The intuition for why this works is that the packets emitted by all non-obfuscated protocols always contain some plaintext headers, whereas randomizers by definition have random data in these locations. They showed their test against obfsproxy achieved a relatively low false-positive rate of only $0.2 \%$ when used against non-obfuscated network traces collected from a university campus. Whether or not this constitutes a sufficiently small false-positive rate in practice is both context-dependent and unclear.

Traffic analysis. Even without parsing packet payloads to exploit the actual byte values being carried, obfuscator-produced traffic may exhibit packet lengths, inter-packet timings and directional traffic patterns that deviate from the background network traffic. Using such meta-data to infer information about communications is referred to as traffic analysis.

One example of deviant traffic behavior arises in Meek. Conventionally, HTTPS connections involve two message flows, one smaller upstream request (client to server), and then a longer downstream message containing the requested content. With Meek, however, the client must frequently send requests to see if new data is available. To a network DPI system, this may stand out as an uncharacteristically large number of upstream messages. The ability to exploit this by a DPI was investigated by Wang et al., who gave a machinelearning-based approach that detected Meek with true positive rate of 0.98 and false positive rate of 0.0002 or less. But their detector may not be easy to use in practice as it only worked well when trained and tested on traces from the same network environment.

For fear of traffic analysis, some obfuscators include mechanisms to try to randomize meta-data such as packet lengths and packet inter-arrival time. Such countermeasures borrow techniques from the extensive literature on website fingerprinting and its countermeasures [9]. How effective these countermeasures are in the obfuscation context is unknown.

\section{Challenges And Open Questions}

So far, we have provided an overview of the rapid evolution of censorship and censorship-evasion technologies in the context of network traffic obfuscation. Now 


\begin{tabular}{|c|c|c|c|c|c|c|}
\hline Obfuscator & Type & Deployment & Key exchange & Perf. & Attacks & Notes \\
\hline Cirripede $[8]$ & tunneling & & $\begin{array}{l}\text { In-band (TLS) with } \\
\text { pre-registration }\end{array}$ & High & & $\begin{array}{l}\text { Requires on-path cooperating } \\
\text { ISP/routers }\end{array}$ \\
\hline CloudTransport $[1]$ & tunneling & & In-band (TLS) & Moderate & & $\begin{array}{l}\text { Google or Amazon HTTPS, cloud } \\
\text { storage as rendezvous }\end{array}$ \\
\hline Collage [2] & mimicry & & $\mathrm{n} / \mathrm{a}$ & Low & & $\begin{array}{l}\text { Embed in user-generated content, } \\
\text { use content servers as rendezvous }\end{array}$ \\
\hline Decoy Routing $\mid 10$ & tunneling & & Out-of-band & High & & Requires on-path cooperating routers \\
\hline Dust 17] & randomizer & uProxy & Out-of-band & High & & $\begin{array}{l}\text { Stream cipher w/ transmitted key } \\
\text { material }\end{array}$ \\
\hline FTE $[11]$ & mimicry & $\begin{array}{l}\text { Lantern, Tor, } \\
\text { uProxy }\end{array}$ & Out-of-band & High & {$[15$} & $\begin{array}{l}\text { Flexible DPI-regex matching of } \\
\text { HTTP, SSH, SMTP, etc. }\end{array}$ \\
\hline Flashproxy $\mid 5$ & tunneling & Tor & $\mathrm{n} / \mathrm{a}$ & Moderate & & $\begin{array}{l}\text { Plaintext tunnel using websockets } \\
\text { through browser-based proxies }\end{array}$ \\
\hline Infranet 4 & mimicry & & In-band private & Low & & $\begin{array}{l}\text { Modulate sequence of HTTP re- } \\
\text { quests to send data }\end{array}$ \\
\hline Marionette 3 & mimicry & & Out-of-band & Moderate & & $\begin{array}{l}\text { Programmable mimicry including } \\
\text { stateful and statistical properties }\end{array}$ \\
\hline $\begin{array}{l}\text { Domain Fronting } \\
\text { (e.g. Meek }[6 \mid \text { ) }\end{array}$ & tunneling & $\begin{array}{l}\text { Tor, Psiphon, } \\
\text { Lantern, } \\
\text { uProxy, } \\
\text { Psiphon, } \\
\text { GoAgent }\end{array}$ & In-band (TLS) & Moderate & {$[15$} & Google or Amazon HTTPS \\
\hline Non-PT Tor & tunneling & & In-band (TLS) & High & {$[18$} & Non-Tor TLS \\
\hline $\operatorname{obfs}\{2,3\} \quad 14$ & randomizer & Tor & Out-of-band & High & {$[15$} & Full encryption of messages \\
\hline obfs4 [14] & randomizer & Tor, Lantern & In-band private & High & {$[15$} & $\begin{array}{l}\text { Uniform key exchange, full encryp- } \\
\text { tion of messages }\end{array}$ \\
\hline ScrambleSuit [19] & randomizer & Tor & In-band private & High & {$[15]$} & $\begin{array}{l}\text { Uniform key exchange, full encryp- } \\
\text { tion of messages }\end{array}$ \\
\hline SkypeMorph [13] & mimicry & & Out-of-band (Skype) & Low & 7 & Mimics Skype connections \\
\hline Stegotorus $\mid 16$ & mimicry & & In-band private & Low & {$[7$} & Mimics HTTP connections \\
\hline Telex 20 & tunneling & & $\begin{array}{l}\text { In-band } \quad \text { (modified } \\
\text { TLS) }\end{array}$ & High & & $\begin{array}{l}\text { Requires on-path cooperating } \\
\text { ISP/routers }\end{array}$ \\
\hline
\end{tabular}

Fig. 4. Comparison of network obfuscation approaches. Types correspond to our classifications of randomizer, mimicry, or tunneling. Any notable deployments are listed. The performance column ("Perf.") is qualitative and based upon numbers reported in the original work.

we turn to the many open questions and challenges that remain. We cluster them into three general areas: understanding users, understanding censors, and futureproofing obfuscation systems. Within each of these areas, we call out specific tasks that need addressing.

\section{A. Understanding Users}

Beyond anecdotes and folklore, very little is understood about the users of censorship-circumvention tools, and this hamstrings the development of effective tools. Research that takes a principled approach to understanding users and their situations could lead to significant improvements. But performing user studies in this context is particularly challenging. The use of censorship-circumvention tools is illegal in some places and, in extreme cases, exposing users could put them in physical danger.

Develop guidelines for user studies. The research community might produce guidelines for performing ethical human subjects research in this space ${ }^{5}$ These guidelines

\footnotetext{
${ }^{5}$ Standard IRB-style human subjects reviews are also important, but could be complemented by further community-driven guidelines.
}

would do well to account for regional and cultural differences, reflect the challenge of obtaining informed consent, and perform measurement in a transparent yet privacy-sensitive way.

Perform user studies. The guidelines could be put to use in the design of ethical research studies. Key questions that might be addressed include: What makes users decide to use a system, or not? How do users first learn of the availability of tools and then obtain them? What are user perceptions regarding risk, ethics, and legality of tool use? How do users react to different kinds of performance or functionality loss when using circumvention tools? How important is it to users that they be able to convincingly deny that they used a tool?

\section{B. Understanding Censors}

Perhaps the most important theme emerging from this paper is that researchers do not yet have a clear understanding of the adversary. This prevents us from knowing whether or not a proposed obfuscation method is truly "broken" or targets an appropriate design trade- 
offs between security, performance, and usability.

Characterize "normal" traffic. Circumvention technologies depend on sensitivity to collateral damage, but at present we lack models for how normal, unobfuscated traffic appears to DPI systems. This is especially important for mimicry-based obfuscators which, by design, try to avoid detection by appearing to be normal traffic.

So far, researchers have either generated synthetic data sets or, in one case [15], gathered traces from a university's networks. The problem with synthetic traces, and even university network captures, is that they are unlikely to properly represent the wide diversity of traffic that censors observe.

One approach here would be to develop partnerships with large network operators (e.g. ISPs) in order to perform studies. We note that for obfuscation research, one unfortunately needs what's referred to as fullpacket capture, i.e. the entire packet contents, including application-layer data. This could have significant privacy implications for the users of the network, so special care is demanded. Perhaps researchers could develop new techniques for doing studies without ever storing packets, and/or incorporate other privacy mechanisms (c.f., [12]). This would improve the privacy of such studies and, one hopes, reduce reluctance of network operators to help perform them.

One powerful artifact of such studies would be useful models of "normal" traffic in various settings. These could be used to build more accurate simulations to support further analysis and tool testing. What would make a model "useful" is not currently well defined.

Models of collateral damage. It seems clear that our threat models must account for the sensitivity of censors to collateral damage. Underlying all censorship circumvention tools is the assumption that censors want to limit collateral damage, at least to some extent. There are a number of open questions here.

First is characterizing what types of collateral damage are of concern to censors. We expect that tolerance to false-positives varies from country to country, and across time within a single country. Moreover, not all collateral damage is the same: it may be that causing collateral damage of certain forms is more costly to censors in terms of political or economic repercussions. Domain fronting systems, such as CloudTransport and Meek both rely on the hope that blocking popular cloud services, e.g. those provided by Google or Amazon, is untenable. However, some countries like China have blocked such services entirely for periods of time. A methodology for systems to reason about collateral damage is clearly needed.

The challenge is for models to incorporate parameters in a way that captures informal statements of the form "the censor will not tolerate more than an $n \%$ falsepositive rate." Few existing works attempt to measure false-positive rates in any sort of methodical manner. Absent of such attempts, we have no way to state that a given technique will induce collateral damage that is intolerable to the modeled censor.

A starting point would be to build threat models parameterized by false-positive rates and standardized methods to identify network traffic. Researchers could then present receiver operating characteristic (ROC) curves with their approach. ROC curves and measures such as area-under-the-curve (AUC) are already standard practice in machine learning, and we recommend bringing these forms of analysis to circumvention technology development.

Accessible censorship adversary labs. Given the widespread and increasing use of commercial DPI devices, good threat models should be informed by what devices can reasonably be expected to do now and in the near future - what computational tasks can they perform (regular expression matching? full parsing of packet payloads? intensive statistical tests?), how much state can they keep (a single packet? all packets in a TCP connection? all connections from a particular IP address?). This assessment will be context-dependent: it will be impacted by the volume of traffic and the assumed sensitivity to collateral damage. This suggest that a hierarchy of threat models should be developed to guide building useful systems, rather than attempting to get consensus on a single model.

A hurdle has been obtaining access to the kinds of equipment used by censors. DPI systems are expensive proprietary devices, and they come with restrictive licensing agreements that typically prevent any benchmarking. This means that researchers, even if they purchase one, may be contractually obligated not to perform research with them. While hardware itself can often be purchased second-hand on sites like Ebay, they may have out-of-date software and not fully reflect the abilities of fully updated deployments.

The community would benefit from a concerted effort to overcome these hurdles, with the goal of building a widely usable censorship lab. The lab would need to obtain realistic, preferably state of the art, devices with realistic configurations. Less restrictive licensing would need to be negotiated. We might look to previous scientific infrastructure projects for inspiration, such as PlanetLab, CloudLab, and the National Cyber Range. We envision such a lab acting as a repository for reference datasets of "normal" traffic (as discussed above), in addition to well providing a battery of existing censorship tools. Ideally, the lab would support remote access for researchers. 


\section{Future-proofing Obfuscation Systems}

We expect that user studies and better understanding of censors will yield significant insights into how to build better circumvention systems. Eventually our goal should be to adapt to, or even defuse, the current armsrace, and give to developers the techniques needed to have future-proof obfuscation mechanisms. By this we mean mechanisms that can maintain connectivity for users even in the face of adaptive censors that know the design of the system. We now summarize the research directions needed to achieve this.

Develop formal foundations for obfuscation research. Currently there is a dearth of formal abstractions formal threat models in particular - upon which to lay principled foundations for obfuscation. One approach is to follow the lead of modern cryptographic theory, where giving precise adversarial models, security goals, and syntax for primitives are primary research considerations. This approach enables clear scientific discussion and objective comparison of systems relative to specified adversarial models. In short, good models are a significant aid in the development of new tools.

Unlike traditional cryptographic problems, where efficient constructions have been shown to meet very pessimistic adversarial models, the censorshipcircumvention setting may be one that benefits from more realistic models, informed and evolved by experimental evidence and measurements from the field.

Build general frameworks. Obfuscation mechanisms have mostly been built with a particular target circumvention system in mind. But obfuscation methods designed for a specific circumvention system may not be easily adapted for another. For example, Tor is TCPbased, so Tor-oriented obfuscation methods need not consider unreliable transports, but I2P and uProxy must.

To have broader impact, the obfuscation community would benefit from general libraries and frameworks. In addition to cutting down on aggregate implementation effort, having broadly useful obfuscation libraries could facilitate rapid rollouts of countermeasures to new attacks - not every tool would need to have a different patch developed. Of course, building moregeneral libraries will require community consensus on typical architectures, APIs, and protocols for negotiating which obfuscation to use.

Increase the use of encryption. Recent efforts to expand the use of standard encryption tools like TLS make it harder to perform censorship. If most of the Internet is end-to-end encrypted using TLS, then censors are harder pressed to use DPI to detect content they wish to block. Indeed we view improved censorship circumvention as a nice ancillary benefit to the already laudable goal of ubiquitous encryption on the Internet.
However, recall our earlier warning that encryption is not a silver bullet for censorship circumvention. Particular applications that use common encryption protocols can still be fingerprinted, often by information sent in the clear such as headers, packet sizes, and timing.

Censors are also sometimes positioned to break endto-end security, performing man-in-the-middle (MitM) attacks either by directly abusing the web's publickey infrastructure (as occurred in Turkey in 2012 $\sqrt{6}$ or by forcing clients to install root certificates that trick systems into allowing MitM proxies. The latter is common in corporate settings already, and retooling those solutions to nation-state levels is part of the threat landscape that should be considered.

Simultaneously, encryption standards could do more to support obfuscation. For example, they could encrypt more of the handshake required during connection establishment. Ideally all bits emitted would be completely random-looking to the censor. (This is targeted by the obfs4 system.) If all TLS connections achieved this then the entropy-based attacks discussed in Section IV] would no longer be effective.

\section{CONCLUSION}

Censorship-circumvention technology is a relatively young field of research but already has a wide diversity of approaches and systems. This suggests the need to reflect and consolidate what has been learned and to propose open questions to facilitate the field's development.

Perhaps the most striking observation is that researchers do not yet have a clear and common model of the adversary. We also noted significant challenges to understanding users and making obfuscation systems robust to future attacks. These limitations are preventing researchers from knowing whether or not a proposed obfuscation method is vulnerable in practice (verses just in theory); and whether a design has made appropriate trade-offs between security, performance, ease of use, or if it is failing to address user needs.

These are open questions in large part due to a surfeit of methodological challenges faced by researchers in this area. Chief among these are the difficulty of obtaining access to network traffic without violating privacy, of procuring DPI systems used by censors, and of performing user studies within relevant populations. Such challenges make principled progress difficult, but also offer opportunities for creative workarounds. We suggested some specific tasks and research directions that the community might take up in order to make progress, including developing guidelines for user studies and building a shared lab for evaluating DPI capabilities.

6 https://googleonlinesecurity.blogspot.com/2013/01/ enhancing-digital-certificate-security.html 
Unfortunately Internet censorship will be around for the foreseeable future. Researchers, activists, and governments supporting the right to unfettered information online should continue to work towards a future in which network traffic obfuscation prevails in the face of sophisticated, well-provisioned censors. This will provide a critical piece to the techno-social puzzle enabling people around the world access to information.

\section{ACKNOWLEDGEMENTS}

The authors would like to thank the participants of the 2014 Obfuscation workshop organized by Google for their valuable contributions.

\section{REFERENCES}

[1] C. Burbaker, A. Houmansadr, and V. Shmatikov. CloudTransport: Using cloud storage for censorship-resistant networking. In Privacy Enhancing Technologies, pages 1-20. Springer, 2014.

[2] S. Burnett, N. Feamster, and S. Vempala. Chipping away at censorship firewalls with user-generated content. In USENIX Security Symposium, pages 463-468. Washington, DC, 2010.

[3] K. P. Dyer, S. E. Coull, and T. Shrimpton. Marionette: A programmable network-traffic obfuscation system. In USENIX Security, August 2015.

[4] N. Feamster, M. Balazinska, G. Harfst, H. Balakrishnan, and D. R. Karger. Infranet: Circumventing web censorship and surveillance. In USENIX Security Symposium, pages 247-262, 2002.

[5] D. Fifield, N. Hardison, J. Ellithorpe, E. Stark, D. Boneh, R. Dingledine, and P. Porras. Evading censorship with browserbased proxies. In Privacy Enhancing Technologies, pages 239258. Springer, 2012.

[6] D. Fifield, C. Lan, R. Hynes, P. Wegmann, and V. Paxson. Blocking-resistant communication through domain fronting. Privacy Enhancing Technologies, 2015(2):1-19, 2015.

[7] A. Houmansadr, C. Brubaker, and V. Shmatikov. The parrot is dead: Observing unobservable network communications. In IEEE Symposium on Security and Privacy, pages 65-79. IEEE, 2013.

[8] A. Houmansadr, G. T. K. Nguyen, M. Caesar, and N. Borisov. Cirripede: Circumvention infrastructure using router redirection with plausible deniability. In ACM conference on Computer and Communications Security, 2011

[9] M. Juarez, S. Afroz, G. Acar, C. Diaz, and R. Greenstadt. A critical evaluation of website fingerprinting attacks. In $A C M$ SIGSAC Conference on Computer and Communications Security, pages 263-274. ACM, 2014.

[10] J. Karlin, D. Ellard, A. Jackson, C. Jones, G. Lauer, D. Mankins, and W. T. Strayer. Decoy routing: Toward unblockable internet communication. Free and Open Communications on the Internet, 2011.

[11] D. Luchaup, K. P. Dyer, S. Jha, T. Ristenpart, and T. Shrimpton. LibFTE: A toolkit for constructing practical, format-abiding encryption schemes. In USENIX Security, August 2014.

[12] A. G. Miklas, S. Saroiu, A. Wolman, and A. D. Brown. Bunker: A privacy-oriented platform for network tracing. In USENIX Symposium on Networked Systems Design and Implementation, pages 29-42, 2009.

[13] H. Mohajeri Moghaddam, B. Li, M. Derakhshani, and I. Goldberg. Skypemorph: Protocol obfuscation for tor bridges. In $A C M$ conference on Computer and communications security, pages 97108. ACM, 2012.

[14] Tor project. Tor pluggable transport. First proposed in 'Proof of concept transport plugin: http headers' available at: https://trac. torproject.org/projects/tor/ticket/2759 with more recent efforts detailed at: https://www.torproject.org/docs/pluggable-transports. html.en 2011.
[15] L. Wang, K. P. Dyer, A. Akella, T. Ristenpart, and T. Shrimpton. Seeing through network-protocol obfuscation. In ACM SIGSAC Conference on Computer and Communications Security, pages 57-69. ACM, 2015.

[16] Z. Weinberg, J. Wang, V. Yegneswaran, L. Briesemeister, S. Cheung, F. Wang, and D. Boneh. Stegotorus: a camouflage proxy for the tor anonymity system. In ACM conference on Computer and communications security, pages 109-120. ACM, 2012.

[17] B. Wiley. Dust: A blocking-resistant internet transport protocol. Technical rep ort. http://blanu. net/Dust. pdf, 2011.

[18] P. Winter and S. Lindskog. How the Great Firewall of China is blocking Tor. In Free and Open Communications on the Internet. USENIX, 2012.

[19] P. Winter, T. Pulls, and J. Fuss. Scramblesuit: A polymorphic network protocol to circumvent censorship. In ACM workshop on Workshop on privacy in the electronic society, pages 213-224. ACM, 2013.

[20] E. Wustrow, S. Wolchok, I. Goldberg, and J. A. Halderman. Telex: Anticensorship in the network infrastructure. In USENIX Security Symposium, 2011. 